
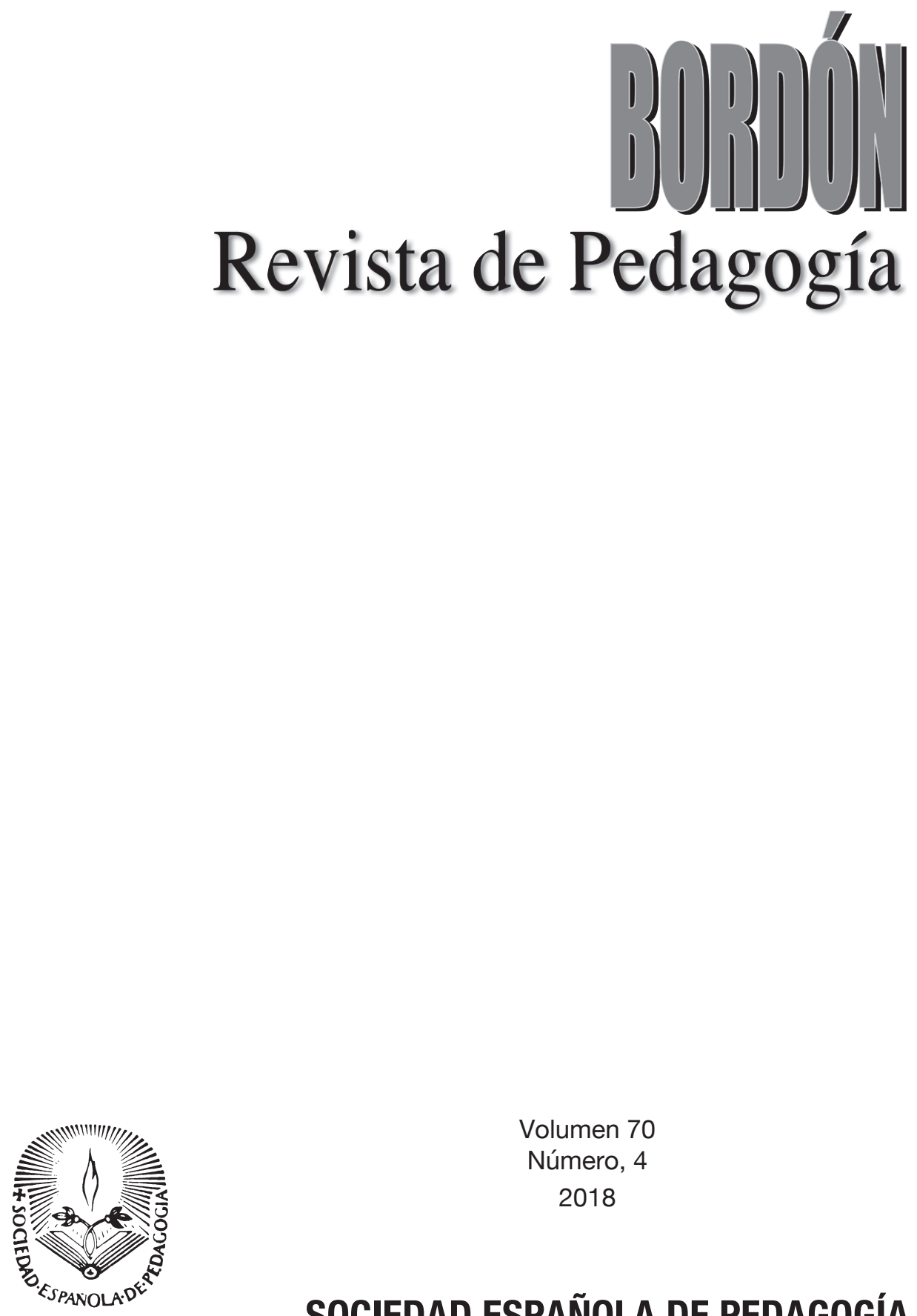

Volumen 70

Número, 4 2018 


\title{
TRAISFORMTACIÓn DEL MODELO EDUCATIUO En EL APRENDIZAJE Y DESARROLLO COMPETEnCIAL. ESTUDIO DE CASO
}

\section{Education Model transformation in regards to learning and competence development. A Case study}

\author{
DÉBORAH MARTÍN R. (1) Y MERCEDES GARCÍA GARCÍA ${ }^{(2)}$ \\ (I) Universidad Internacional de La Rioja \\ (2) Universidad Complutense de Madrid
}

DOI: 10.13042/Bordon.2018.60992

Fecha de recepción: 09/1I/2017 - Fecha de aceptación: 10/09/2018

Autora de contacto / Corresponding author: Déborah Martín R. E-mail: info@pedagogiaparaelexito.com

INTRODUCCIÓN. El artículo describe el efecto que tiene en el alumnado una experiencia de transformación de un centro hacia un modelo versátil que traslada al aula el uso de metodologías activas y colaborativas. Se realiza en un entorno real, a través del asesoramiento de una consultora externa, durante dos cursos. El objetivo es valorar el desarrollo competencial, la satisfacción y el rendimiento académico de los estudiantes en función de los cambios observados en tres aulas. MÉTODO. Estudio de caso. Se describe el contexto, la información previa y los cambios observados tras la transformación. Se realizan entrevistas grupales, análisis documental de las memorias del centro y la comparación de las calificaciones académicas mediante análisis. RESULTADO. La transformación a lo largo de dos años se manifiesta en tres modelos educativos de distinta versatilidad que muestra diferencia en los resultados del alumnado. En el aula que se transforma hacia una mayor versatilidad y en la que se incorporan técnicas didácticas variadas y activas se percibe una evolución favorable en el desarrollo de la competencia lingüística, matemática, social y cívica, una mejora en el comportamiento y rendimiento, y una mayor satisfacción del alumnado con la organización del aula, el aprendizaje por proyectos, la participación en su aprendizaje y la apertura al entorno. Por el contrario, no se aprecia mejora donde la transformación no se produce. DISCUSIÓN. Los resultados coinciden con investigaciones previas sobre el impacto de las culturas versátiles de los centros y aulas en el aprendizaje y clima escolar. Las mejoras se observan cuando el modelo educativo se desarrolla a lo largo del tiempo, al menos dos cursos, apoyado por el liderazgo pedagógico y en entorno educativo. Muestra nuevas líneas de investigación sobre los efectos que las técnicas ligadas a la versatilidad tienen en el desarrollo competencial del alumnado pero también en la competencia docente y en la calidad de los centros educativos.

Palabras clave: Cambio organizativo, Cambio educativo, Aprendizaje activo, Satisfacción, Estudio de caso. 


\section{Introducción}

La necesidad de adecuar las escuelas para dar respuesta a las demandas sociales y económicas actuales supone flexibilizar las organizaciones (Dede, 2007; Yelland, Kalantzis y Cope, 2008; Partnership21, 2007; ITE, 2010; Looney y Michel, 2014). Este proceso conocido como organización educativa versátil debe asumir, según Martín-Moreno Cerrillo (2006), cambios para: personalizar los procesos educativos; diseñar con flexibilización la organización del espacio, tiempos, materiales y recursos; promover un sentido de comunidad y conexión del conocimiento, mediante trabajo en equipo, integración de contenidos, interrelación de estructuras y agrupamientos flexibles; dinamizar mediante un buen liderazgo pedagógico que fomente la participación del profesorado, la familia y el alumnado en la toma de decisiones; abrirse al entorno estimulando la utilización escolar de los recursos; fomentar el bienestar y un entorno amable, donde todos se sientan acogidos.

En definitiva, el cambio de modelo educativo exige no solo un cambio de metodología educativa sino un cambio más global, replanteando el ambiente y la organización del aula y del centro para que desarrolle una cultura versátil que proporcione situaciones diversas de aprendizaje, espacios para la reflexión y el intercambio e incluir la evaluación continua para tomar decisiones de mejora permanente (Looney y Michel, 2014).

La cultura versátil de un centro se proyecta en el aula y, por consiguiente, repercute en el aprendizaje de los estudiantes. El aula de la organización versátil fomenta la diferenciación de espacios, los grupos de trabajo cambiantes y las técnicas didácticas activas para promover la autonomía y la responsabilidad del estudiante y el aprendizaje compartido e interrelacionado entre diferentes áreas de conocimiento (López Martínez, 2005) y también las ganas de estar, convivir y aprender. De hecho, las metodologías activas, inductivas y las técnicas didácticas de investigación, como el aprendizaje basado en proyectos
(ABP), en problemas o retos (ABPr), los debates y las asambleas, o el aprendizaje colaborativo parecen tener un mayor impacto en el rendimiento, en las habilidades cognitivas y de procesamiento del conocimiento (Bietenbeck, 2014; Méndez Martínez, 2015) y así es percibido por los estudiantes (Bietenbeck, 2014; Rappolt-Schlichtmann, Daley y Rose, 2012; Murillo, 2008; Hattie, 2009, 2012; Looney y Michel, 2014; Martínez y McGrath, 2016). Todo esto lleva a pensar que el docente y los equipos educativos de un centro deben aprender nuevas formas de trabajo e incorporar métodos de enseñanza y técnicas didácticas orientadas al aprendizaje profundo y a las necesidades personales del alumnado. Se requiere una transformación en la gestión del aula y en la organización educativa del centro hacia una cultura versátil, un proceso de cambio que necesita de una planificación previa y una ejecución progresiva compartida en la comunidad escolar.

Es un proceso complejo que debe liderar el equipo directivo o un asesor pedagógico para promover la participación activa y colaborativa, el cambio de estrategias educativas, la apertura al entorno, etc. Si bien no se produce al mismo tiempo ni de la misma manera en cada aula o en cada docente. Por consiguiente, una escuela que acepta iniciar un proceso de transformación hacia una organización versátil pero con diferentes resistencias, ¿ ipuede mejorar de la misma manera el aprendizaje de los estudiantes? ¿Puede ser diferente en función del grado de implicación docente en el cambio? ¿Es satisfactorio el cambio?

Este artículo trata de responder a estas cuestiones a través de la situación de partida y evolución hacia la versatilidad de un centro de difícil desempeño de la Comunidad de Castilla-La Mancha.

\section{Método}

\section{Objetivos}

El objetivo de este trabajo se centra en valorar el rendimiento, el desarrollo competencial y la 
satisfacción de los estudiantes en función del tiempo que han estado en un proceso de enseñanza-aprendizaje activo durante la transformación de un centro en una organización versátil. Como la transformación organizativa tiene un impacto diferente en cada docente y por tanto en cada aula hace cuestionarse el efecto que pudiera tener en el aprendizaje y resultados de los estudiantes. Se parte del supuesto de que una organización versátil y el uso frecuente de metodologías activas, mejora el aprendizaje y satisfacción del alumnado. En concreto, se trata de identificar las competencias que, según el profesorado, se han desarrollado en el alumnado al incorporar metodologías activas y técnicas didácticas versátiles; la satisfacción del alumnado con el cambio y su percepción de las técnicas didácticas que mejoran su aprendizaje y la posible mejora del rendimiento académico en función de cada grupo.

\section{Metodología y unidad de análisis}

El estudio de un proceso de transformación educativo tiene que realizarse en su entorno real y utilizar la información de los directamente implicados como fuente de conocimiento. Por ello, se plantea un estudio de caso único.

La unidad de análisis es un centro de educación infantil y primaria de titularidad pública en la Comunidad de Castilla-La Mancha tipificado como centro de difícil desempeño, cuyo equipo directivo tiene interés en iniciar un cambio tras los bajos resultados de su alumnado en las pruebas de Conocimientos y Destrezas Indispensables para Educación Primaria (CDI). Tras los primeros contactos y entrevistas se decide iniciar el proceso de transformación en tres grupos de primaria, $3^{\circ}, 4^{\circ}$ y $5^{\circ}$ (grupo A, grupo B y grupo C, respectivamente) compuesto en su conjunto por 31 estudiantes y sus correspondientes docentes.
El proceso del centro hacia una organización versátil y el cambio metodológico observado en las tres aulas objeto de análisis es dinamizado por una asesora pedagógica externa durante dos años.

Durante los dos cursos escolares se realizan semanalmente grupos de trabajo y reflexión pedagógica con la asesora. La participación en estos encuentros es voluntaria, pero se acuerda que las decisiones tomadas deben llevarse a cabo en toda la organización. Durante las sesiones de trabajo se realiza formación en técnicas didácticas activas, en programación por competencias y en evaluación por estándares de aprendizaje siguiendo el currículo de la Ley Orgánica para la Mejora de la Calidad Educativa (LOMCE, 2013). Cada docente traslada y pone en práctica en su aula lo que se va reflexionando y aprendiendo en el grupo de reflexión, pero respetando su estilo y recursos.

Durante este tiempo se producen incidencias no previstas En el grupo A, la profesora deberá ser sustituida durante el segundo curso escolar.

En definitiva, a lo largo de los dos cursos se refleja la idiosincrasia de cada aula y se registran las circunstancias ocurridas a lo largo de la transformación versátil en los tres grupos del centro objeto de estudio (Martín Rodríguez, 2017, resumidas en la tabla 1 ).

\section{Variables e instrumentos}

La transformación organizacional y metodológica no es objeto de este trabajo aunque tras el proceso observado y resumido en la tabla 1 se infieren tres modelos educativos de diferente versatilidad: el grupo A de organización y metodología más estática; el grupo B de organización y metodología parcialmente versátil; y el grupo C de organización y metodología versátil.

Las variables relacionadas con los efectos esperados en los estudiantes son: 
1. Desarrollo competencial del alumnado según la percepción del profesorado. Se realiza el análisis documental de dos memorias anuales del centro, y, al finalizar, son presentadas a Inspección.

2. Satisfacción del alumnado con el cambio y su percepción de las metodologías y técnicas didácticas utilizadas en el aula que mejoran su aprendizaje, a través de entrevistas grupales en cada uno de los grupos por separado.
3. Rendimiento académico, calificaciones obtenidas antes de iniciar la transformación y al finalizar, de cada uno de los tres grupos.

Las fuentes de información son los propios implicados en el proceso, docentes y estudiantes, a través de los instrumentos utilizados en los centros como procedimientos de evaluación y entrevistas semiestructuradas grabadas para el análisis posterior. Se convocó a las familias a entrevistas grupales, pero se

TABLA 1. Características en la transformación del aula de los grupos A, B y C

\begin{tabular}{llll}
\multirow{2}{*}{ Características } & \multicolumn{3}{c}{ Aula } \\
\cline { 2 - 4 } & Grupo A & Grupo B & Grupo C \\
\hline
\end{tabular}

En el $1^{\circ}$ curso escolar se producen cambios en la dirección planificada. El

Evolución proceso se detiene por baja de la profesora. En el $2^{\circ}$ curso el profesor suplente mantiene un modelo docente centrado en la enseñanza

$1^{\circ}$ curso: trabajo por Técnicas didácticas incorporadas proyecto, interdisciplinar. $2^{\circ}$ curso: técnica expositiva
Estable y progresiva hacia un modelo versátil centrado en el alumno
Estable, progresiva, rápida y completa transformación $\begin{array}{ll}\text { del docente y } & \\ \text { libro de texto }\end{array}$

\section{del docente y ejercicios del}

\begin{tabular}{|c|c|c|}
\hline Agrupamientos & Individual o parejas & $\begin{array}{l}\text { Agrupamientos } \\
\text { heterogéneos }\end{array}$ \\
\hline
\end{tabular}

Disposición de mesas de forma individual o parejas, generalmente mirando a la pizarra y al docente.

Organización espacial Muy ocasionalmente las del aula mesas se colocan en forma de U.

El aula no tiene espacios versátiles para realizar tareas diferenciales

La disposición de las mesas se organiza, generalmente, en equipos de 4 o 3 pero varía en función de las actividades. No se organiza el espacio de manera versátil para realizar tareas diferenciales
Aprendizaje basado en proyectos, debates, asambleas, trabajo colaborativo con roles, y experimentos
Aprendizaje basado en proyecto, interdisciplinar, trabajo por parejas, lectura de estudio en el aula y aclaración de dudas con la profesora, debates, utilización de porfolio. Opción de subir nota al superar la asignatura

Agrupamientos heterogéneos u homogéneos

Aula versátil: espacios de

En el centro, las salidas aumentan considerablemente: al inicio, dos salidas (memoria inicial

Entorno del centro) y durante el proceso se llegan a realizar 13 salidas por curso.

$\mathrm{Al}$ inicio, 4 visitas de profesionales al centro y durante el proceso se amplían a 8 
desestimó la información considerándola no representativa por su baja asistencia.

\section{Procedimiento de recogida y análisis de datos}

Durante los primeros días del inicio de curso, se toma nota de los resultados de las pruebas de CDI realizado al grupo A, y los resultados académicos obtenidos en los 3 grupos. Se analiza el contenido de la memoria anual de centro que había sido entregada a la Administración educativa antes de iniciar el proceso de cambio.

Este estudio defiende los principios de complementariedad metodológica, utilizando métodos cualitativos y cuantitativos para describir y comprender la realidad del proceso y resultados (Klafki, 1988: 100). El análisis cualitativo se realiza mediante el programa Atlas.Ti.8.00 en el que se estudia el contenido de las memorias del centro y las entrevistas grupales realizadas al alumnado. La codificación que se lleva a cabo es descriptiva, deductiva y abierta. El análisis presenta la percepción que tiene el profesorado del nivel competencial de los estudiantes antes y después, las modificaciones realizadas en las aulas y la satisfacción del alumnado.

A modo exploratorio, se analizan los resultados académicos mediante análisis descriptivo y la Prueba de Rangos de Wilcoxon con SPSS23.0, comparando las puntuaciones de cada grupo antes y después de la transformación.

\section{Resultados}

Se presentan los resultados teniendo en cuenta cada grupo en desarrollo competencial, satisfacción y rendimiento.

\section{Desarrollo competencial}

La memoria del centro antes de empezar el proceso relata:
Tras las pruebas CDI pudimos comprobar que realmente hay un problema principalmente en la adquisición de las competencias lingüística, matemática, de aprender a aprender y autonomía e iniciativa personal.

Algunos alumnos además tienen problemas para desarrollar su competencia emocional. En general, en nuestro centro la competencia lingüística se ve afectada principalmente por la lengua materna de los alumnos, su nivel de adquisición de castellano y por el nivel sociocultural de las familias. La competencia matemática se ha visto influida principalmente por dificultades de atención por parte de los alumnos, que debido a falta de hábitos no están acostumbrados a mantener la concentración en materias académicas durante el tiempo que se necesita para resolver un problema. Creemos que sería necesario trabajar la atención de alguna manera en nuestros alumnos, aunque también esto se debería trabajar en casa con la colaboración de las familias. También muchos necesitan realizar un programa de técnicas de estudio, desarrollar la autonomía personal y aprender a aprender (memoria inicial).

El análisis del contenido de la memoria inicial del centro, según se resume en la figura 1 , describe al alumnado en general con bajas competencias en todos los ámbitos y dificultades para el aprendizaje relacionadas con conductas disruptivas, falta de atención y escucha, y el no respeto de los turnos de palabra, hablar en voz baja, o seguir instrucciones, además de baja autoestima, interés y autonomía.

\section{Grupo A}

El proceso de este grupo le ha llevado, durante el segundo año, a volver a una situación organizativa y metodológica más estática, centrada en el docente, similar a la que tenía el centro al inicio.

En la memoria final del grupo se relata:

Tenían problemas de concentración y de falta de interés por lo académico. En algunos momentos 
Figura 1. Diagrama de codificación de las características del alumnado del centro en general antes del proceso de transformación

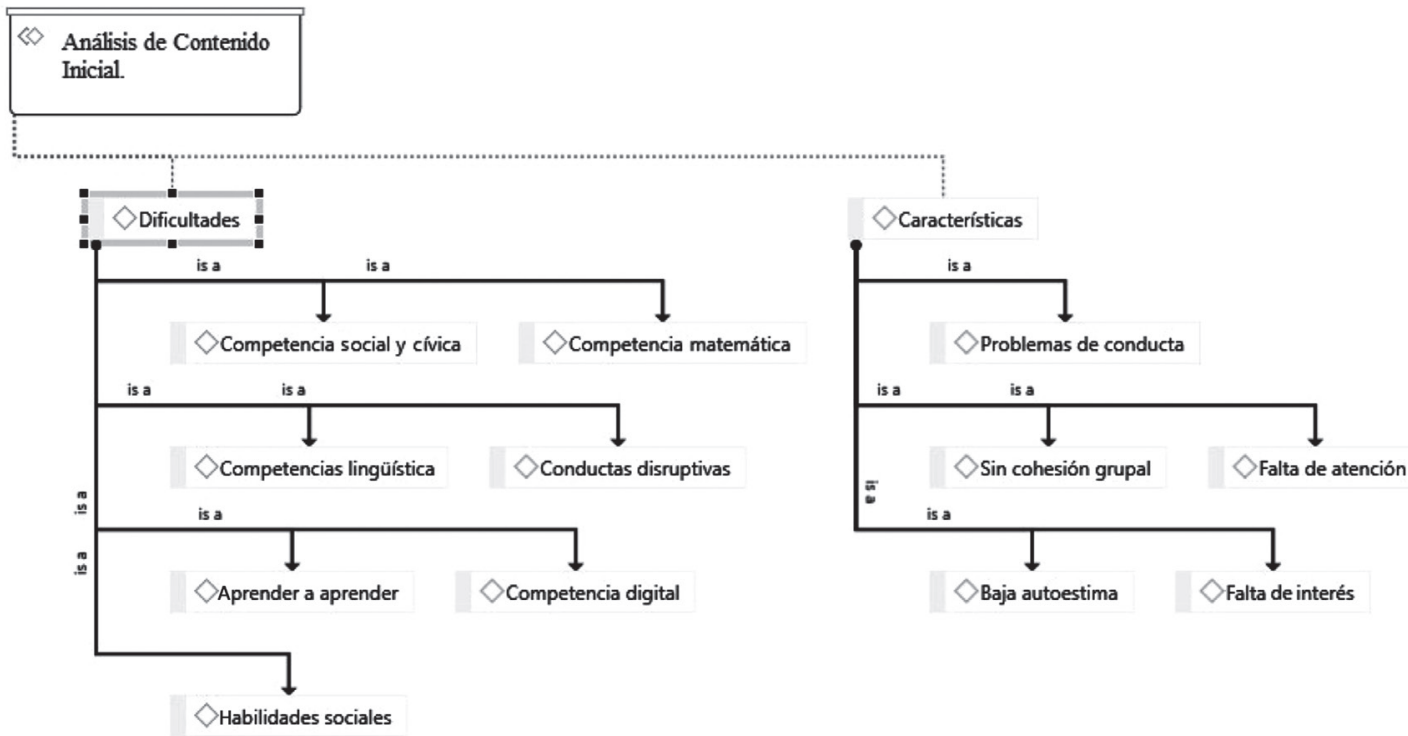

incluso se han podido observar muchas conductas disruptivas que han impedido el funcionamiento normal de la clase (memoria final).

El análisis del contenido de la memoria final, según se resume en la figura 2, no recoge ninguna valoración sobre el desarrollo competencial del alumnado del grupo A, pero sí un incremento de conductas disruptivas, baja autoestima, desmotivación o falta de interés por lo académico y un

Figura 2. Diagrama de características que incrementan en el grupo A tras el proceso de transformación

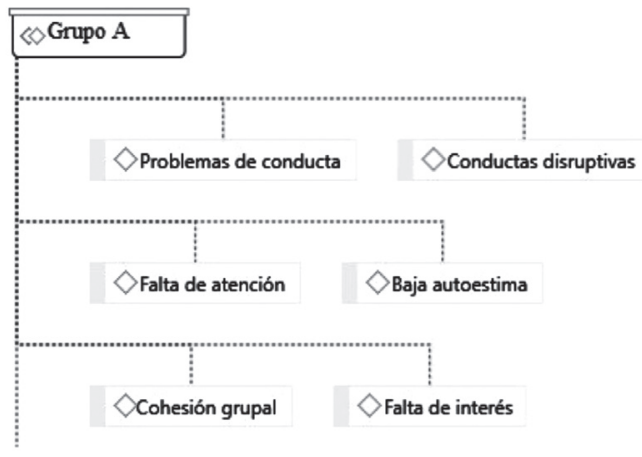

clima de aula complicado. Este grupo es descrito de forma semejante al alumnado de inicio del proceso.

\section{Grupo B}

El proceso del grupo B le ha llevado al final del periodo de transformación a una organización del aula más versátil, con agrupamientos homogéneos y trabajo colaborativo aunque mejorable en la disposición diferenciada del espacio.

En la memoria final del grupo se relata:

Se han mejorado las competencias lingüística, digital, matemática y la social y cívica, trabajando con distintas metodologías — trabajo en parejas, en equipo, en gran grupo, individual, investigación, memorización, trabajo por tareas y proyectos, etc. [...].

En los momentos de discusión y de pelea se razonaba la situación con todos los alumnos del grupo. Se analizaban las distintas situaciones de conflicto y se llegaba a un acuerdo. Al final 
el grupo ha funcionado bien como unidad y los alumnos que al principio se encontraban más excluidos al principio han encontrado su lugar dentro del grupo-clase [...].

Grupo socialmente cohesionado, con una motivación intrínseca bastante alta en actividades de clase, sobre todo, con ordenador, tareas manipulativas y trabajos en grupo y por parejas. Es más complicado hacerles trabajar en casa - alrededor de un tercio no suelen traer los deberes-. Los dos alumnos de NEE han trabajado muy bien, y están muy bien integrados en el grupo (memoria final).

El análisis de contenido de la memoria final, resumido en la figura 3, describe una mejora de las competencias lingüística (aunque todavía insuficiente en algunos aspectos relevantes para la comprensión de los contenidos), digital, matemática y social, según el profesorado. Además, muestra una buena cohesión grupal y motivación en las actividades de clase, sobre todo cuando se trabaja con ordenador, tareas manipulativas y en grupo o en parejas. Se ha desarrollado la autoestima, las habilidades sociales y la cohesión grupal. No obstante, aunque han mejorado, las habilidades sociales siguen siendo una dificultad y precisan de mayor trabajo.

El profesorado del grupo B considera que los resultados de ciencias sociales y ciencias naturales no han sido del todo satisfactorios debido a que el grupo trabaja con el nuevo currículo de la LOMCE para Castilla-La Mancha, cuyos libros de textos han aumentado el vocabulario técnico:

Las mayores dificultades del grupo han sido en Lengua, CC. Sociales y CC. Naturales, donde necesitan mayor competencia lingüística, sobre todo en los aspectos de vocabulario, comprensión y velocidad lectora. Aunque se ha leído mucho - y les gusta leer en clase todo tipo de libros y textos- necesitan leer mucho más. Han evolucionado bastante desde principios de curso. Necesitan seguir en esa línea de trabajos en grupo y

FIGURA 3. Diagrama de competencias y características que incrementan en el grupo B tras el proceso de transformación

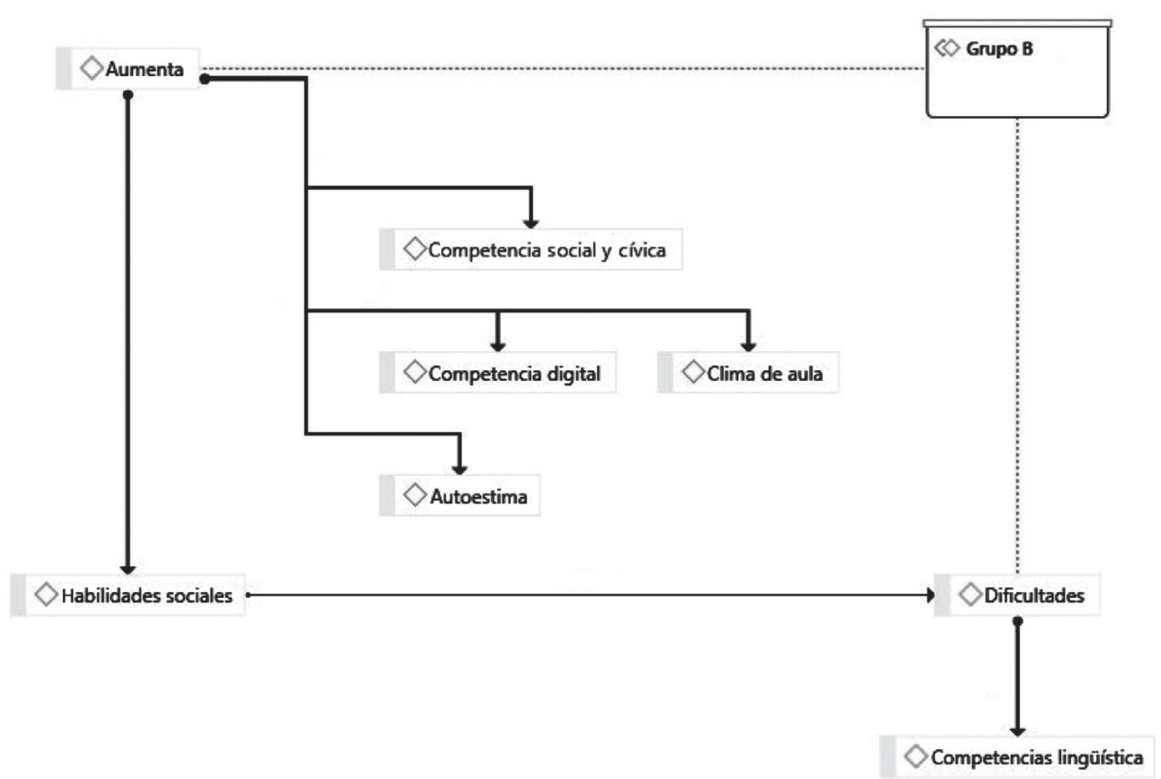


por parejas, actividades de clase - intentando no dejar muchos deberes, o que los deberes sean leer o estudiar - y tareas manipulativas. Necesitan leer muchos textos variados, manejar el diccionario y hacer presentaciones orales de sus propios trabajos para desarrollar la competencia lingüística de manera adecuada (memoria final).

\section{Grupo C}

El proceso de este grupo le ha llevado a convertirse en un aula versátil.

En la memoria inicial del grupo se relata:

Grupo con variedad de niveles, muy inquietos, es necesario ser firme para centrar su atención en el estudio. Dos alumnos de etnia gitana han presentado a lo largo del curso problemas de conducta y de reconocimiento de la autoridad. Un número alto de alumnos necesitan un programa de autoestima para favorecer su integración social (memoria inicial).
Mientras que en la memoria final:

Grupo heterogéneo pero muy motivado en general, demandante de trabajos, proyectos y tareas. Tenía cinco alumnos en riesgo social, uno de ellos, repetidora de este curso - que al final aprueba todo-y los otros cuatro promocionan (memoria final).

Han avanzado muchísimo en general. Además, se ha fomentado la unión del grupo completo, $y$ aunque han tenido algunos problemas de relación (algún alumno con otro), en su mayor parte se han solucionado de manera fácil por el propio grupo (memoria final).

El alumno es capaz de evaluar su propia conducta (memoria final).

El análisis de contenido de las memorias muestra que es un grupo que pasa de tener problemas con la autoridad, muy baja autoestima y baja atención, según manifiesta el profesorado en la

FIgURA 4. Diagrama de competencias y características que incrementan en el grupo C tras el proceso de transformación

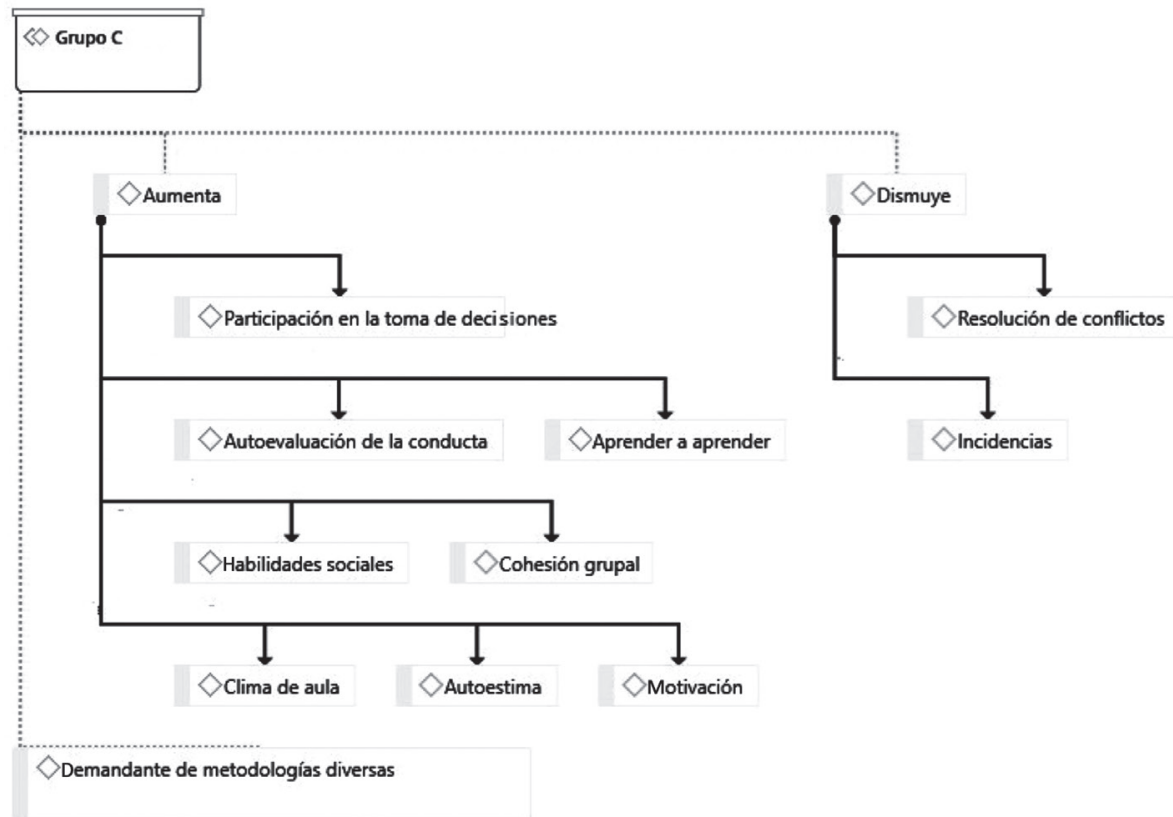


fase inicial, a ser un grupo cohesionado, resumido muy demandante de metodologías diversas, como proyectos, trabajos, parejas, exposiciones orales, etc. (figura 4). El profesorado manifiesta también que las mayores dificultades que presentan los alumnos son en asignaturas relacionadas con las competencias lingüísticas: lengua, inglés y francés, posiblemente porque 10 alumnos son de nacionalidad extranjera.

Por otra parte, se observa la disminución de la gravedad de incidencias y la mejora del clima del aula. Se destaca la cohesión grupal y la participación de los alumnos en la toma de decisiones para solucionar los conflictos. Además se incrementa la autoevaluación y la reflexión de la propia conducta.

En definitiva, parece que el desarrollo competencial puede ser diferente según el proceso de transformación del docente y su correspondiente aula. Mientras que en un aula estática el profesorado no recoge información sobre las competencias del alumnado pero refleja problemas de conducta y falta de interés, en el aula que introduce una organización y cultura versátil, parece que mejoran las capacidades y características del alumnado positivas para el aprendizaje y clima escolar.

\section{Satisfacción}

La satisfacción del alumnado con la metodología y las técnicas didácticas asociadas a la transformación se analiza en cada uno de los tres grupos:

\section{Grupo A}

Este grupo expresa que no ha trabajado en equipo el segundo año, pero que sí lo hizo el curso anterior cuando estaban con su tutora. Por otra parte, sobre la forma de trabajo recuerdan un proyecto interdisciplinar de "Caballeros" (historia, lengua, educación física y música), del que dicen haber aprendido un montón de cosas. También el proyecto de autogestión "Mercadillo Solidario", en el que participó todo el centro.

A la pregunta de si han percibido cambios en el aula o en la manera de dar las clases expresan que el cambio ha sido el profesor, alguno menciona a la tutora anterior (echándola de menos). Afirman que el profesor actual les pone muchos deberes y es muy estricto.

Sobre la organización de espacios, prefieren trabajar en forma de U frente a estar sentados de manera individual, que es como suele estar habitualmente.

Alumno: Porque podemos hablar con todos. Alumna: Porque podía ver mejor la pizarra. Alumna: Porque veo delante.

Alumna: También porque había más espacio. Alumno: Se veía todo.

Comentan que les gustaría trabajar más con los ordenadores y que hubiera más tiempo de descanso entre clases. Es decir, en vez de un recreo largo varios recreos cortos. No expresan ninguna otra actividad o estrategia que recuerden les haya hecho aprender.

\section{Grupo B}

En la entrevista los estudiantes del grupo B valoran muy positivamente trabajar en equipo y no trabajar tanto con el libro, exámenes de test cada cierto tiempo y tener pocos deberes para casa. Ante la petición de propuestas para el curso siguiente teniendo en cuenta las estrategias que más les han ayudado, dicen: el trabajo en equipo, hacer otro proyecto de "mercadillo", trabajar más con los ordenadores y hacer menos deberes.

Asesor: ¿Qué os gustaría seguir haciendo el año que viene?

Estudiantes: Trabajar en equipo.

Estudiantes: Hacer otro mercadillo, pero mejor, para ir a otra excursión. 


\section{Grupo C}

Los estudiantes del grupo C expresan en la entrevista qué les ha resultado de mayor interés y les ha facilitado el aprendizaje: la organización del aula con varias zonas, una zona con rincones temáticos donde traer elementos diferentes sobre el tema quincenal, debates, exposiciones al resto de compañeros y leer los temas en clase de manera individual o por parejas. También la realización de los viernes de debate de noticias sociales y el rincón con portfolios.

Han trabajado mucho la comprensión lectora y, antes de cada tema, la profesora les pide que primero lean en clase y luego, sobre lo leído, se explica y aclaran las dudas. Según comentan, esta técnica les ha ayudado a aprender más. También les ha gustado mucho que los exámenes fueran diferentes y que, algunas veces, coevaluación o la oportunidad de subir nota cuando han aprobado. El alumnado de este grupo muestra agradecimiento hacia su profesora durante la entrevista.

Ante la propuesta de aportar sugerencias y mejoras, manifiestan que prefieren seguir trabajando así y que les gustaría tener educación física y educación plástica todos los días.

En la memoria final también se relata la observación del profesorado sobre la satisfacción del grupo y su actitud positiva hacia el aprendizaje:

Nos encontramos un grupo muy motivado, sobre todo, para realizar trabajos en grupo y por parejas. Demandaban tareas constantemente a la tutora y a otros profesores, haciendo pósteres, redacciones y exponiendo los trabajos oralmente (memoria . Evaluación final).

\section{Rendimiento}

Las notas académicas de los tres grupos se han comparado antes y después de la transformación. En primer lugar, se describen los grupos a partir de las medidas de tendencia central, media y mediana, y la amplitud intercuartílica, y después se analizan las diferencias de rendimiento de cada grupo.

\section{Grupo A}

La media del rendimiento académico del grupo A en la fase inicial es de 7,0111 (mediana, 7) mientras que en la fase posterior obtiene una media de 5,8667 (mediana, 5,70). El 50\% del grupo se sitúa entre la puntuación 6 y 7,80 en la fase previa y entre 5 y 7,2 al finalizar. En la figura 5 se observa una mayor heterogeneidad en las notas obtenidas al final del proceso. El 25\%

Figura 5. Diagrama de caja y bigotes del rendimiento del grupo A en su contexto inicial y posterior
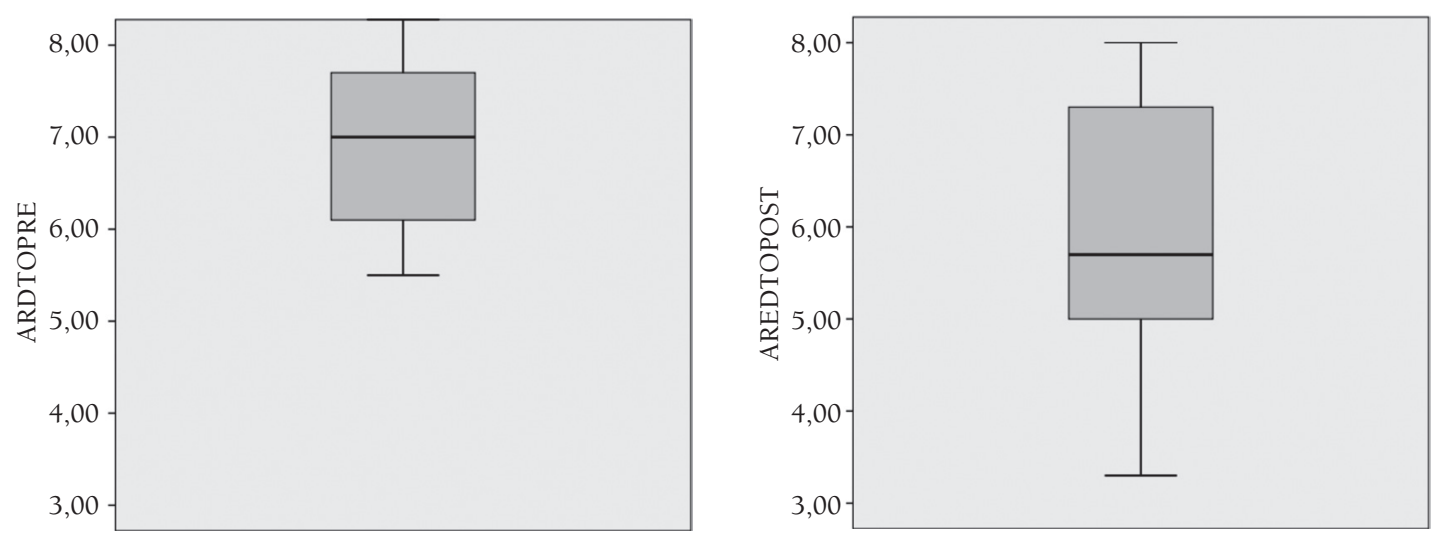
inferior se sitúa entre los 3 y 5 puntos mientras que al inicio estaba entre 5,5 y 6 puntos.

En la memoria final el profesorado manifiesta que el grupo A obtiene un nivel académico muy bajo con pocos hábitos de estudio y lectura, además de los ya citados problemas de disciplina:

Grupo difícil, con un rendimiento escolar muy bajo de media. Hay 10 alumnos que suspenden conocimiento del medio, y 6 matemáticas, y la educación artística también está en un nivel muy bajo - aunque nadie suspende- - La mayor parte del alumnado se encuentra entre el suficiente y el bien en casi todas las áreas. No tienen hábitos de estudio ni de lectura en su casa, y en las últimas semanas de curso ha habido algunos problemas de disciplina. La actitud de los padres tampoco favorece, más bien al contrario. Repite un alumno, con cuatro áreas suspensas (memoria final).

\section{Grupo B}

La media del rendimiento académico del grupo B en su contexto inicial es de 7,66 (mediana, 7,70 ) y en el contexto final es similar, 7,22 (mediana, 7,60). En la figura 6 se observa que el 50\% del grupo se distribuye entre las puntuaciones 6,10 y 8,50 , mientras que es algo más heterogéneo al finalizar, entre las puntuaciones 5,50 a 8,10 . Por otra parte, el $25 \%$ de la situación final se sitúa entre un 4,60 y 5,50 (figura 6), menor que antes del proceso.

\section{Grupo C}

La media de rendimiento del grupo $C$ en su contexto inicial es de 5,95 puntos (mediana, 5,70 ) y la media posterior es de 6,69 (mediana, $6,20)$. El 50\% del grupo se distribuye entre las puntuaciones 5,50 y 7,50 al inicio, y entre 5,70 y 8,50 al finalizar, observándose un rango intercuartílico mayor pero con una puntuación mínima por encima del aprobado. Mientras que el $25 \%$ del grupo al finalizar se sitúa por encima de 8 puntos, esta es la puntuación máxima antes de la transformación. El 25\% inferior en la fase posterior se sitúa entre 5,00 y 5,70 puntos, inferior a lo que sucede al inicio del proceso (figura 7).

Las diferencias de rendimiento observadas antes y después de la transformación en cada uno de los grupos docentes se comparan mediante la prueba de Wilcoxon.

En la tabla 2, los rangos negativos indican una mayor puntuación en el pretest que en el postest; los positivos, que en el postest es mayor que en el pretest; y los empates señalan que no hay diferencia entre las puntuaciones.

Figura 6. Diagrama de caja y bigotes del rendimiento del grupo $B$ en su contexto inicial y posterior
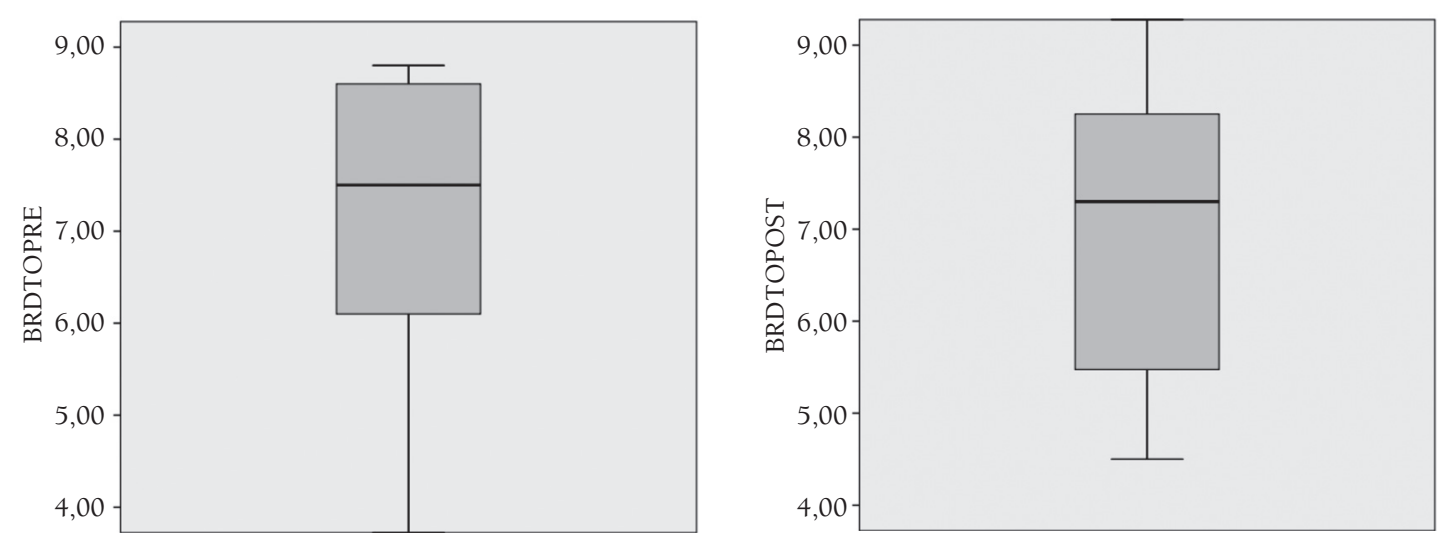
Figura 7. Diagrama de caja y bigotes del rendimiento del grupo $C$ en su contexto inicial y posterior
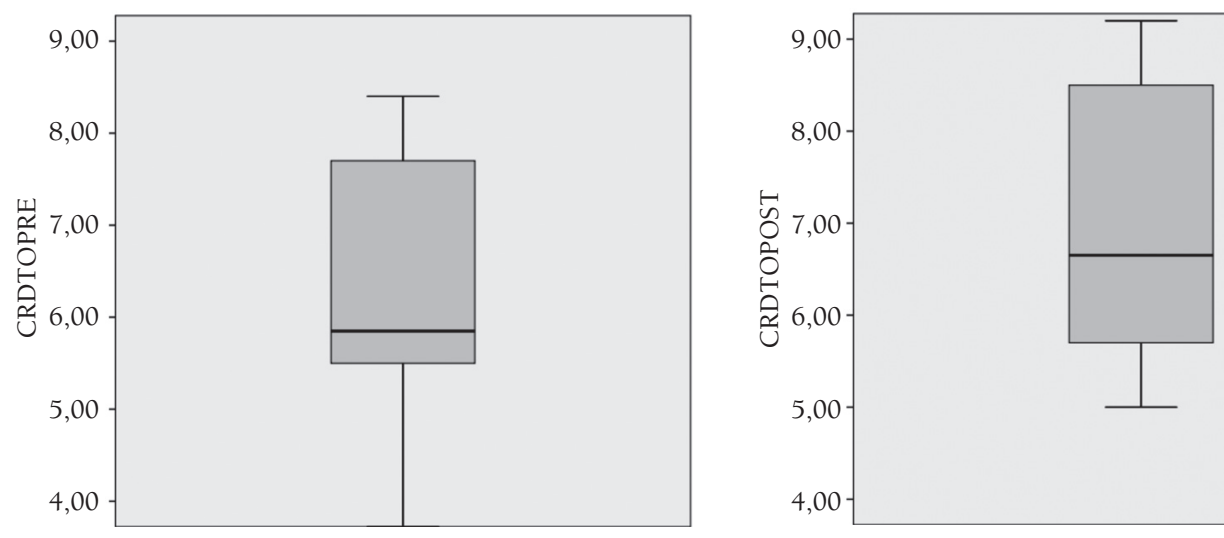

TABla 2. Rangos obtenidos en rendimiento para cada grupo en la prueba de Wilcoxon

A RDTOPOST - RDTOPRE

\begin{tabular}{lrrr} 
Negativos & 9 & 5,00 & 45,00 \\
\hline Positivos & 0 &, 00 &, 00 \\
\hline Empates & 0 & & \\
\hline Total & 9 & 7,75 & 31,00 \\
\hline Negativos & 4 & 4,00 & 24,00 \\
\hline Positivos & 6 & & \\
\hline Empates & 2 & & 3,00 \\
\hline Total & 12 & 3,00 & \\
\hline Negativos & 1 & 5,25 & \\
\hline Positivos & 8 & & \\
\hline Empates & 1 & & \\
\hline Total & 10 & & \\
\hline
\end{tabular}

En la tabla 3, a un nivel de significación de 0,05 se observan diferencias significativas en el grupo $\mathrm{A}(\mathrm{p}=0,008)$ y en el grupo $\mathrm{C}(\mathrm{p}=0,021)$ mientras que no hay diferencias en el rendimiento del grupo $B(p=0,720)$. Estas diferencias son a favor del pretest en el grupo A y a favor del postest en el grupo C. Es decir, parece que en el grupo C se observa una mejora del rendimiento tras el

TABla 3. Resultados de la prueba de Wilcoxon en rendimiento para cada grupo

Grupo

\begin{tabular}{rrr} 
A & $-2,670$ &, 008 \\
\hline B &,- 3580 &, 720 \\
\hline C & $-2,310$ &, 021
\end{tabular}

Rdto.Post-Rdto.Pre
Sig. asintótica (bilateral) 
proceso de transformación, mientras que en el grupo A, el rendimiento es menor.

\section{Discusión y conclusiones}

En este trabajo se reflexiona sobre los efectos percibidos de las organizaciones versátiles que suponen una mayor flexibilidad en los procesos educativos y el uso de metodologías activas, colaborativas y abiertas al entorno. Los procesos de transformación escolar son complejos. La planificación educativa y el diseño de una investigación pueden verse modificados en un sistema como el español caracterizado por su rigidez, la inestabilidad de su plantilla, y la falta de espacios y tiempos para la reflexión y desarrollo profesional del equipo. En un mismo centro implicado en un proceso de transformación, aunque todo el profesorado participe en las sesiones formativas, como ha sido el caso de este centro, no siempre se traduce de la misma manera en el aula. En consecuencia, el alumnado de un centro puede participar de procesos educativos distintos. Los estudios previos ponen de manifiesto que la versatilidad de una organización diseñada para el desarrollo de competencias necesarias en el siglo XXI requiere del apoyo de la dirección o de un líder pedagógico pero también la implicación del profesorado para introducir metodologías flexibles.

A lo largo de dos años que dura el proceso de cambio se han observado diferentes niveles de transformación en las aulas. Frente a un aula que inicia la transformación y se interrumpe, otras avanzan hacia organizaciones versátiles. En estas últimas parece que hay una mayor probabilidad de favorecer el desarrollo competencial de los estudiantes (lingüístico, matemático, social y cívico), la motivación por aprender, la satisfacción, el rendimiento académico, resultados similares a los estudios de Bietenbeck (2014), Méndez Martínez (2015), Hattie (2009), Murillo (2006, 2008), López López (2009) y De los Cobos, Gómez, Gómez Pérez y Gómez (2011).
Pero no solo se perciben mejoras relacionadas con el aprendizaje, también en conducta y en la cohesión grupal, como en López Alacid (2009) y Palacios (2007) o en autonomía y autoestima (Bietenbeck, 2014; Rappolt-Schlichtmann, Daley y Rose, 2012; Murillo, 2008; Hattie, 2009, 2012; Looney y Michel, 2014). Por el contrario, en el grupo donde se interrumpe el proceso de trasformación, se percibe un aumento de incidencias y conductas disruptivas.

Es interesante también la opinión del alumnado de este estudio que dice tener más ganas de trabajar en el centro, recuerda mejor lo aprendido y aprende mejor a través del ABP, similar al informe KeyCoNet (2014) o los estudios de Hattie (2009), Murillo (2008) y López López (2009) y también afirma que lo que más le ayuda a aprender y le gusta es: cambiar la estructura física del aula con rincones temáticos y diferenciados, porque se pueden mover; cambiar la disposición de las mesas porque se ven todos, ven mejor la pizarra y pueden hablar entre ellos; trabajar en equipo; realizar debates; leer en clase el contenido de estudio para profundizar en los textos y resolver o plantear dudas con la profesora; trabajar por proyectos; tener exámenes "diferentes" que no sean de memorizar; el portfolio; coevaluarse; tener la opción de subir nota cuando han superado la asignatura; tener menos deberes para casa porque los realizan en clase; utilizar otros materiales que no sean solo el libro de texto; trabajar con los ordenadores; y hacer proyectos abiertos al exterior, como el mercadillo donde implicaron a personas de fuera del centro, asumieron una responsabilidad y se pudieron ir de excursión gracias a su esfuerzo.

La investigación educativa de campo está llena de imprevistos que impiden controlar variables relevantes, esta dificultad se incrementa cuando se desarrolla un estudio longitudinal. La unidad muestral que aceptó participar en el estudio, un centro pequeño de difícil desempeño, se caracteriza, además, por la inestabilidad del profesorado y los cambios frecuentes del alumnado. En 
consecuencia, se presenta el estudio de un caso que permite, a lo largo de dos años, explorar en un entorno real para acercar las teorías a la práctica, interpretar y comprender aunque no se pueda generalizar. Por otra parte, se pone en valor la percepción de los participantes y el uso de los documentos elaborados en el centro para analizar la realidad, siendo conscientes de que son medidas subjetivas y que, sumado a una muestra pequeña, permiten análisis menos potentes. Aun así, los resultados resaltan la importancia de las metodologías en el aprendizaje y la conducta de los estudiantes, y animan a pensar que el cambio docente es posible aunque para que perdure es necesario que la comunidad educativa, la dirección del centro y la Administración educativa se impliquen y, sobre todo, que la mejora no sea efecto de intervenciones puntuales ni tan siquiera de un curso escolar; sino que requiere de, al menos, dos cursos o, para que sea un cambio de cultura y metodología realmente efectivo, de 3 a 5 años (Fullan, 1997, 2016). Los datos nos advierten de la necesidad de estudiar longitudinalmente el efecto de las metodologías activas y participativas no solo en el aprendizaje y en el rendimiento, también en la cohesión y comportamiento de los estudiantes.

En definitiva, el estudio muestra los cambios planificados en un entorno real con rigor en el tratamiento y análisis de los datos y trata de mostrar el impacto percibido que tienen los modelos versátiles, nos muestra la dirección y da pie a formular hipótesis de una línea de investigación prometedora para evaluar los cambios en el aprendizaje de los estudiantes, en la competencia docente y en la calidad de los centros educativos:

Las escuelas que dan un primer paso para dirigirse hacia un modelo versátil, centrado en el aprendizaje y orientado al desarrollo competencial, comenzarán a manifestar mejoras significativas en la calidad de aprendizaje, la participación de los estudiantes y el desarrollo de sus competencias se incrementarán (Pace, 2013: 5-6).

\section{Referencias bibliográficas}

Bietenbeck, J. (2014). Teaching practices and cognitive skills. Labour Economics, 30, 143-153.

Consejo Escolar del Estado (2015). Informe sobre el estado y situación del sistema educativo: curso 2013-2014. Madrid: Ministerio de Educación y Deporte.

Dede, C. (2007). Introduction: A sea change in thinking, knowing, learning, and teaching. En G. Salaway y J. Borreson Caruso (eds.), The ECAR study of undergraduate students and information technology (pp. 19-26). Boulder. Colorado: EDUCAUSE.

De los Cobos, A., Gómez, M., Gómez, J., Pérez, M. y Gómez, J. (2011). Relación entre la aplicación de metodologías docentes activas y el aprendizaje del estudiante universitario. Bordón. Revista de Pedagogía, 63(2), 27-40.

Fullan, M. (1997). The Challenge of School Change: A Collection of Articles. IRI/Skylight Training and Publishing.

Fullan, M. (2016). La dirección escolar: Tres claves para maximizar su impacto. Madrid: Ediciones Morata.

Hattie, J. (2009). Visible Learning: A Synthesis of Over 800 Meta-Analyses Relating to Achievement. Nueva York: Routledge.

Hattie, J. (2012).Visible learning for teachers. Nueva York: Routledge.

ITE (2010). Habilidades y competencias del siglo XXI para los aprendices del milenio en los países de la OCDE. Madrid: Instituto de Técnicas Educativas.

Klafki, W. (1988). ¿Se modifica la realidad escolar por efectos de la investigación escolar? Revista de Educación, 286, 97-113. 
Ley Orgánica 2/2006, de 3 de mayo, de Educación. Publicada en el BOE el 4 de mayo de 2006.

Looney, J. y Michel, A. (2014). KeyCoNet's Conclusions and Recommendations for Strengthening Key Competence Development in Policy and Practice. Final Report. Bruselas: European Schoolnet.

López Alacid, M. P. (2009). Efectos del aprendizaje cooperativo en las habilidades sociales, la educación intercultural y la violencia escolar: un estudio bibliométrico de 1997 a 2007. Alicante: Biblioteca Virtual Miguel de Cervantes.

López López, E. (2009). Evaluación del efecto de variables críticas en el aprendizaje de los escolares. ESE. Estudios Sobre Educación, 16, 55-78.

López Martínez, A. (2005). La organización del espacio en los centros educativos: un factor determinante para el cambio de las estructuras organizativas. Bordón. Revista de Pedagogía, 57(4), 519-533.

Martín Rodríguez, D. (2017). La organización educativa versátil y el desarrollo competencial (tesis doctoral inédita). Madrid: Universidad Complutense de Madrid.

Martín-Moreno Cerrillo, Q. (2006). Organización y dirección de centros educativos innovadores. El Centro Educativo Versátil. Madrid: McGraw-Hill.

Martínez, M. R. y McGrath, D. (2016). Deeper learning: How eight innovative public schools are transforming education in the twenty-first century. Nueva York: The New Press.

Méndez Martínez, I. (2015). Prácticas docentes y rendimiento estudiantil. Evidencia a partir de TALIS 2013 y PISA 2012. Madrid: Fundación Santillana.

Murillo, F. J. (2006). Una Dirección Escolar para el Cambio: del Liderazgo Transformacional al Liderazgo Distribuido. REICE. Revista Electrónica Iberoamericana sobre Calidad, Eficacia y Cambio en Educación, 4(4), 11-24.

Murillo, F. J. (2008). Hacia un modelo de eficacia escolar. Estudio multinivel sobre los factores de eficacia de las escuelas españolas. REICE. Revista Electrónica Iberoamericana sobre Calidad, Eficacia y Cambio en Educación, 6(1), 4-28.

Pace, L. (2013). Competency Education Series: Policy Brief One an Emerging Federal Role for Competency Education. Cincinnati, Ohio: Knowledgeswork.

Palacios, S. G. (2007). Requisitos para una inclusión de calidad en el tratamiento educativo de la diversidad. Bordón. Revista de Pedagogía, 59(4), 581-595.

Partnership 21 (2007). Framework for 21st century skills. Washington: P21.

Rappolt-Schlichtmann, G., Daley, S. G. y Rose, L. T. (eds.) (2012). A Research Reader in Universal Design for Learning. Cambridge, MA: Harvard Education Press.

Yelland, N., Cope, B. y Kalantzis, M. (2008). Learning by Desing: creating pedagogical frameworks for knowledge building in the twenty-first century. Asia-Pacific Journal of Teacher Education, 36(3), 197-213.

\section{Abstract}

Education Model transformation in regards to learning and competence development. A Case study

INTRODUCTION. This paper discusses the effect on students of an experience of transformation from a static school into a versatile model that transfers to the classroom the use of active and collaborative methodologies. It is carried out in a real environment, through the advice of an external consultant, during two courses. The aim is to assess the development of competence, satisfaction and academic performance of the students according to the changes observed after the transformation. METHOD. The research design a case study. It describes the context, the 
information before to and the changes observed after the transformation. Information is collected by group interviews, documentary analysis of reports and analysis academic achievement. The treatment of the data has been through qualitative and quantitative analysis. RESULTS. The transformation over two years is manifested in three educational models of different versatility. Teacher's perception come out the effect that versatile models centred on learning have on the competence development of students, specifically linguistic, maths, social and civic competence, an improvement in behaviour and performance and greater student satisfaction with the organisation of the classroom, learning by projects, participation in learning and openness to the environment. On the other hand, there is no improvement where the transformation has not taken place. DISCUSSION. The results are coincident with research as European reports on the impact of versatile school and classroom cultures on learning and school climate. In addition, improvements are observed when the educational model is developed over time, at least two courses, supported by pedagogical leadership. It shows new lines of research on the effects that techniques linked to versatility have on the development of students'skills but also on teaching skills and the quality of schools.

Keywords: Organizational change, Educational change, Active learning, Satisfaction, Case studies.

\section{Résumé}

La transformation du modele educative d'apprentissage et development de competences.

Étude d'un cas

INTRODUCTION. Larticle décrit l'effet sur les élèves d'une expérience de transformation méthodologique d'un centre vers un modèle polyvalent, basée sur l'utilisation de méthodologies actives et collaboratives. L'expérience a été mis en place dans un environnement réel, avec l'aide et les conseils d'un consultant externe, pendant deux années scolaires. L'objectif a été d'évaluer le développement des compétences, la satisfaction et la performance académique des élèves dans trois salles de classe. METHODE. Étude de cas. On y décrit le contexte, les informations précédentes et les changements observés lors la transformation de la méthode éducative. On a effectué des entretiens de groupe, l'étude documentaire des mémoires du centre ainsi qu'une comparaison à travers une analyse spécifique des résultats académiques des élèves. RÉSULTAT. La transformation après deux années se montre par trois modèles éducatifs de versatilité différente qui reflètent aussi des différences dans les résultats des élèves. Le groupe transformé vers une polyvalence plus importante et sur lequel on a appliqué de techniques didactiques variées et actives montre une évolution favorable dans le développement des compétences linguistiques, mathématiques, sociales et civiques ainsi qu'une amélioration du comportement et de la performance académique. On a pu observer que les élèves sont plus satisfaits avec la nouvelle façon d'organiser les cours, par rapport à l'apprentissage par projets, la prise d'un rôle actif dans les processus d'apprentissage et l'ouverture à l'environnement. Au contraire, on a pu constater qu'il n'y a pas d'amélioration là où il n'y a pas eu de changements, DISCUSSION. Les résultats coincident avec des recherches précédentes sur l'impact du climat scolaire et les cultures polyvalentes dans les écoles et les salles de classe. On peut y constater l'amélioration lorsque le modèle éducatif est développé, au fil du temps, au moins pendant deux années scolaires, soutenus par l'encadrement pédagogique et l'environnement éducatif. Larticle nous montre de nouvelles lignes de recherche à propos des effets des techniques liées à la polyvalence et le développement 
des compétences sur les élèves mais aussi sur le niveau de compétences pédagogiques des professeurs et sur la qualité pédagogique des centres de formation.

Mots-clés: Changement éducatif, Changement organisationnel, Apprentissage actif, Satisfaction, Étude de cas.

\section{Perfil professional de las autoras}

\section{Déborah Martín R. (autora de contacto)}

Doctora en Educación. Máster en Innovación e investigación educativa. Pedagoga, psicóloga forense y criminóloga. Directora de Centros Socio Educativos. Miembro de dos grupos de investigación "Pedagogía adaptativa" de UCM y "Metodologías Activas y Mastery Learning" de UNIR. Actualmente es directora de la Escuela UNIR de Formación de Profesores en Tecnología Educativa y Competencias. Su línea de investigación se relaciona con la transformación de las organizaciones educativas hacia metodologías activas y el desarrollo competencial. Consultora y formadora en Pedagogía para el Éxito

Correo electrónico de contacto: info@pedagogiaparaelexito.com

Dirección para la correspondencia: UNIR. Av. de la Paz, 137, 26004 Logroño, La Rioja

\section{Mercedes García-García}

Doctora en Ciencias de la Educación. Profesora titular de Pedagogía Diferencial del Departamento de Investigación y Psicología en Educación de la Facultad de Educación de la Universidad Complutense de Madrid. Codirectora del grupo de investigación UCM-940424 "Pedagogía Adaptativa y codirectora del Proyecto I+D+i EDU2015-63844-R PROFICIENCyIn+E" (MINECO/FEDER). Sus líneas de investigación giran en torno a: teoría y estrategias adaptativas en contextos escolares; evaluación y desarrollo de competencias docentes; y evaluación de competencias genéricas en la universidad.

Correo electrónico de contacto: dr.diversidad@ucm.es 